OPEN ACCESS

Edited by:

Shengtao Zhou,

Sichuan University, China

Reviewed by:

Xiuxing Wang,

Nanjing Medical University, China

Yuhong Zhou,

Fudan University, China

Zhe Li,

Fudan University, China

Houbao Liu,

Fudan University, China

*Correspondence:

Chuan Wang

chuanwangfmu@163.com

${ }^{\text {t}}$ These authors have contributed equally to this work

Specialty section: This article was submitted to Women's Cancer, a section of the journal

Frontiers in Oncology

Received: 17 November 2020 Accepted: 06 April 2021

Published: 03 May 2021

Citation:

Guo W, Li J, Huang H, Fu F, Lin Y and Wang C (2021) LnCRNA PCIR Is an

Oncogenic Driver via Strengthen the

Binding of TAB3 and PABPC4

in Triple Negative Breast Cancer.

Front. Oncol. 11:630300.

doi: 10.3389/fonc.2021.630300

\section{LncRNA PCIR Is an Oncogenic Driver via Strengthen the Binding of TAB3 and PABPC4 in Triple Negative Breast Cancer}

\author{
Wenhui Guo ${ }^{1,2,3 \dagger}$, Jingyi $\mathrm{Li}^{4 \dagger}$, Haobo Huang ${ }^{5 \dagger}$, Fangmeng $\mathrm{Fu}^{1,2,3 \dagger}$, Yuxiang $\mathrm{Lin}^{1,2,3}$ \\ and Chuan Wang ${ }^{1,2,3 *}$
}

${ }^{1}$ Department of Breast Surgery, Fujian Medical University Union Hospital, Fuzhou, China, ${ }^{2}$ Department of General Surgery, Fujian Medical University Union Hospital, Fuzhou, China, ${ }^{3}$ Breast Cancer Institute, Fujian Medical University, Fuzhou, China, ${ }^{4}$ College of Integrated Traditional Chinese and Western Medicine, Fujian University of Traditional Chinese Medicine, Fuzhou, China, ${ }^{5}$ Department of Blood Transfusion, Fujian Medical University Union Hospital, Fuzhou, China

Long non-coding RNAs (LnCRNA) as the key regulators in all stages of tumorigenesis and metastasis. However, the underlying mechanisms are largely unknown. Here, we report a IncRNA RP11-214F16.8, which renamed Lnc-PCIR, is upregulated and higher RNA level of Lnc-PCIR was positively correlated to the poor survival of patients with triple negative breast cancer (TNBC) tissues. Lnc-PCIR overexpression significantly promoted cell proliferation, migration, and invasion in vitro and in vivo. RNA pulldown, RNA immunoprecipitation (RIP) and RNA transcriptome sequencing technology (RNA-seq) was performed to identify the associated proteins and related signaling pathways. Mechanistically, higher Lnc-PCIR level of blocks PABPC4 proteasome-dependent ubiquitination degradation; stable and highly expressed PABPC4 can further increase the stability of TAB3 mRNA, meanwhile, overexpression of Lnc-PCIR can disrupt the binding status of TAB3 and TAB2 which lead to activate the TNF- $\alpha / N F-\kappa B$ pathway in TNBC cells. Our findings suggest that Lnc-PCIR promotes tumor growth and metastasis via up-regulating the mRNA/protein level of TAB3 and PABPC4, activating TNF- $\alpha / N F-\kappa B$ signaling pathway in TNBC.

Keywords: long non-coding RNA, RP11-214F16.8/Lnc-PCIR, TNF- $\alpha$ /NF-KB signaling pathway, TGF-beta activated kinase 1 (MAP3K7) binding protein 3, poly(A) binding protein cytoplasmic 4

\section{BACKGROUND}

Breast cancer is the most frequently diagnosed malignancy in women worldwide and is the second leading cause of cancer-related death in the United States $(1,2)$. Expression of the estrogen receptor (ER), progesterone receptor (PR), and amplification of the HER2 gene define the main breast cancer subtypes in terms of prognostic and therapeutic intervention (3). Triple negative breast cancer (TNBC) is the breast cancer subtype characterized by the absence of expression of the ER, PR and 
HER2 (4).Therapies commonly used in other breast cancer subtypes are therefore not suitable for TNBC, and treatment options are largely limited to conventional genotoxic chemotherapy $(5,6)$. Most of TNBC patients present high rates of metastatic recurrence and very poor long-term prognosis after chemotherapy. Consequently, to explore the underlying mechanisms and identification of molecular targets and the development of new therapeutic avenues remain critically important.

Long non-coding RNAs (lncRNAs) are a class of transcripts longer than 200 nucleotides lacking the open reading frame with no protein-coding ability (7). With advancements in cancer transcriptome profiling, large number of lncRNAs has been demonstrated closely associated with cancer $(8,9)$. Besides, lncRNAs have also been implicated to regulate a range of biological functions, such as genomic imprinting and transcriptional regulation, plays a critical role in tumorigenesis and metastasis (10-12). During the past decade, numerous studies have showed the important role of NF- $\kappa \mathrm{B}$ pathway as a link between inflammation and tumorigenesis (13). NF- $\kappa \mathrm{B}$ was first identified as part of the immune system, and become widely accepted as a crucial transcription factor that regulates inflammation, innate and adaptive immunity, cell proliferation, cell differentiation and apoptosis $(14,15)$. The hyper-activate of $\mathrm{NF}-\kappa \mathrm{B}$ pathway has been linked to cancer which is regarded as a potential therapeutic target in human cancers $(16,17)$. TGF-beta activated kinase 1 (MAP3K7) binding protein 3 (TAB3), as a newly identified Transforming growth factor- $\beta$-activated kinase 1 (TAK1) binding partner, has been implicated in the immune response, signal transduction, inflammation and autophagy (18-21). Several reports showed TAB3 is markedly overexpressed in various tumor tissues, such as the testis, skin, non-small cell lung cancer (NSCLC), hepatocellular carcinoma (HCC) and small intestinal cancers (19, 22-24). However, the mechanism of overexpressed $\mathrm{TAB} 3$ in the process of TNBC remains unclear.

In this study, we sought to identify clinically relevant lncRNAs deregulated specifically in TNBC patients and aim to reveal the functional role and regulatory mechanism of lncRNA in the progress of TNBC. We identified and characterized the lncRNA RP11-214F16.8, renamed Lnc-PCIR (LncRNA Positively Correlated with Inflammatory Responses). Our results showed Lnc-PCIR was upregulated in TNBC tissues, and overexpressed of Lnc-PCIR could accelerated cell growth and metastasis in vitro and in vivo. For mechanistic investigations, Lnc-PCIR could

\footnotetext{
Abbreviations: LncRNA, Long non-coding RNA; TNBC, Triple Negative Breast Cancer; Lnc-PCIR, LncRNA Positively Correlated with Inflammatory Responses; CCK-8, Cell Counting Kit-8; RIP, RNA Immunoprecipitation; TCGA, The Cancer Genome Atlas; TAB3, TGF-beta activated kinase 1 (MAP3K7) binding protein 3; PABPC4, poly(A) binding protein cytoplasmic 4; TAB2, TGF-beta activated kinase 1 (MAP3K7) binding protein 2; UCSC, University of California, Santa Cruz; qPCR, quantitative real-time PCR; RACE, rapid amplification of cDNA ends; MS, mass spectrometry; ER, estrogen receptor; $\mathrm{PR}$, progesterone receptor; TAK1, Transforming growth factor- $\beta$-activated kinase 1 ; NSCLC, non-small cell lung cancer; HCC, hepatocellular carcinoma; GSEA, Gene Set Enrichment Analysis; GO analysis, Gene Ontology Analysis; EMT, Epithelial-Mesenchymal Transition; CHX, cycloheximide.
}

directly binding with $\mathrm{TAB} 3$ and PABPC4. The stability of PABPC4 protein was increased by inhibiting ubiquitin proteasome degradation and overexpressed PABPC4 can enhance the stability of TAB3 mRNA, which known as a key molecular involved in TNF- $\alpha / \mathrm{NF}-\kappa \mathrm{B}$ signaling pathway. Taken together, these results suggest that Lnc-PCIR may be as the promising therapeutic target for TNBC patients.

\section{METHODS}

\section{Cell Culture}

Human TNBC cell lines used in this study were purchased from Cell Bank of the Chinese Academy of Science (Shanghai, China), the Health Science Research Resources Bank (Osaka, Japan) and American Type Culture Collection (ATCC, Manassas, Virginia, USA). The cell lines were cultured in Dulbecco's modified Eagle's medium (DMEM, Gibco BRL) contained with $10 \%$ fetal calf serum (FBS, HyClone) as well as $100 \mathrm{U} / \mathrm{ml}$ penicillin and $100 \mu \mathrm{g} / \mathrm{ml}$ streptomycin (Invitrogen). Cells were maintained in a humidified incubator at $37^{\circ} \mathrm{C}$ in the presence of $5 \% \mathrm{CO} 2$. Cell lines confirmed to be mycoplasma-free by short tandem repeats (STR) profiling. All the cell lines were used within 15 passages and subjected to routine cell line quality examinations (e.g., morphology, mycoplasma), and thawed fresh every 2 months.

\section{Patients and Samples}

Cohort 1: One hundred and ten paired TNBC and neighboring noncancerous tissues from TCGA database (https:// cancergenome.nih.gov/); Cohort 2: Five hundred and fifty paired patients TNBC and neighboring noncancerous tissues were also from TCGA database; Cohort 3: One hundred and ten paired TNBC and neighboring noncancerous tissues obtained from the surgical specimen archives of the Shanghai cancer center of Fudan university, Shanghai, China (collected postoperatively from August 2010 to September 2018); Each sample was snap-frozen in liquid nitrogen and stored at $-80^{\circ} \mathrm{C}$ prior to RNA isolation and $\mathrm{qRT}-\mathrm{PCR}$ analysis. All patients recruited to this study did not receive any pre-operative treatments. The data do not contain any information that could identify the patients. All patients provided written informed consent. The use of human clinical specimens in the present study was approved by the Institutional Review Board of the Shanghai Medical College of Fudan University. A summary of the clinical information for the 110 patients is available online in Supplementary Table 1.

\section{RNA Isolation and Quantitative PCR (Real-Time RT-PCR)}

Total RNA was extracted from tissues or cultured cells using TRIzol reagent (Invitrogen). Total RNA (500 ng) was reverse transcribed to cDNA in a final volume of $10 \mu \mathrm{l}$ using random primers under standard conditions with the PrimeScript RT Reagent Kit (Takara, Dalian, China). We performed real-time PCR analyses using SYBR Premix Ex Taq (Takara) according to 
the manufacturer's instructions. Results were normalized to the expression of glyceraldehyde 3-phosphate dehydrogenase (GAPDH) or $\beta$-actin, and data were collected based on the comparative cycle threshold (CT) $\left(2^{-\Delta \Delta C T}\right)$ method. Specific primer sequences are listed in Supplementary Table 2.

\section{Plasmid Generation and Transfection/Infection}

We obtained the full-length Lnc-PCIR, PABPC4 and TAB3 sequence and ligated into the pcDNA3.0 (+) vector (Invitrogen). Additionally, we also cloned the sequence of PABPC4 and TAB3 into the pCMV-Flag or pCMV-HA. Plasmid were transfected into TNBC cells cultured in six-well plates using the Lipofectamine 3000 DNA transfection reagent (life, USA). For lentivirus packaging, $2 \times 10^{6} 293 \mathrm{~T}$ cells were co-transfected with packaging plasmid pA2 and pMD2G. After $8 \mathrm{~h}$, the transfection medium was replaced with fresh DMEM supplemented with $10 \%$ FBS. Subsequently, cell supernatants containing lentiviruses were collected. For infection, cells were treated with polybrene (8 $\mu \mathrm{g} / \mathrm{mL}$, Sigma) for $0.5 \mathrm{~h}$ before incubated with lentiviral, after incubation for 8 to $12 \mathrm{~h}$ at $37^{\circ} \mathrm{C}$, stable cell lines were selected by puromycin ( $2 \mu \mathrm{g} / \mathrm{mL}$, Sigma). Cells were harvested for qRT-PCR or western blot analysis $48 \mathrm{~h}$ after transfection. The sequences for the gene-specific primers used are listed in Supplementary Table 2.

\section{Northern Blot Assay}

Total RNA $(10-15 \mu \mathrm{g})$ from samples were separated on $15 \%$ denaturing polyacrylamide gels, transferred onto GeneScreen Plus membranes (PerkinElmer), and hybridized using UltraHyb-Oligo buffer (Ambion). Following hybridization at $42^{\circ} \mathrm{C}$ overnight, the membranes washed twice in $0.1 \times$ SSPE and $0.1 \%$ SDS at $42^{\circ} \mathrm{C}$ for $15 \mathrm{~min}$ each. Membranes were then exposed to a storage phosphor screen (GE Healthcare BioSciences) for $8 \mathrm{~h}$ and imaged using a Typhoon 9410 Variable Mode Imager (GE Healthcare Bio-Sciences). The sequences for probe primers used are listed in Supplementary Table 2.

\section{Western Blotting}

Cells were lysed in lysis buffer in the presence of protease inhibitor cocktail (Roche) and phosphatase inhibitor cocktails I and II (Sigma). Equal amounts of protein, as determined by the Bradford assay, were resolved by electrophoresis in a SDS $10 \%$ polyacrylamide gel and then transferred to a PVDF membrane (Millipore). The membrane was incubated with the primary antibodies and secondary antibodies, then was detected using an enhanced chemiluminescence kit (ECL, Invitrogen). The details of antibodies used are listed in Supplementary Table 3.

\section{Cell Growth Assay}

For cell growth assays, 2000 cells per well were seeded into 96well plates, with three wells used for each group. Cell numbers were evaluated for 5 days using a cell counting kit-8 (CCK-8) (Dojindo, Japan). $10 \mu \mathrm{l}$ of CCK-8 reagent was added to each well and the plate was incubated at $37^{\circ} \mathrm{C}$ for $2 \mathrm{~h}$. Next, the absorbance at $450 \mathrm{~nm}$ was measured in each well by using a spectrophotometer (Molecular Devices, CA, USA). For the colony formation assay, 1500 cells well were seed into 6-well plates and routinely cultured for 14 days. The cells were subsequently fixed with $30 \%$ formaldehyde for $10 \mathrm{~min}$ and stained with $0.1 \%$ crystal violet for $10 \mathrm{~min}$. The number of colonies was determined under an optical microscope.

\section{Cell Migration and Invasion Assays}

The migration and invasive ability of the cells was performed using a transwell assay. For Invasion assays, we using the $8-\mu \mathrm{m}$ pore inserts Millicell chambers which were coated with $30 \mu \mathrm{g}$ of Matrigel (BD Biosciences, USA). And the Millicell chambers without coating the Matrigel was conducted in the migration assay. Cells $(5 \times 10$ (4) for migration and $1 \times 10$ (5) for invasion) were seeded onto a transwell plate with 8 - $\mathrm{mm}$ pores, and DMEM supplemented with $20 \%$ FBS was used as a chemoattractant. Following a 24-h incubation, non-invading cells were manually removed using a cotton swab. Subsequently, the cells were fixed in $4 \%$ paraformaldehyde for $20 \mathrm{~min}$, stained with hematoxylin and then counted under a microscope.

\section{RNA Pull-Down and Mass Spectrometry Assay}

A full-length of sense and antisense Lnc-PCIR sequence were transcribed and biotin-labeled using T7 RNA polymerase (Roche, Basel, Switzerland) and purified using the RNA Clean Kit (ZYMO research, USA) in vitro. $1 \mathrm{mg}$ protein lysis of 231 cell extracts was then mixed with $2 \mu \mathrm{g}$ of biotinylated RNA at $4^{\circ} \mathrm{C}$ for $1 \mathrm{~h}$, and then incubated with $50 \mu \mathrm{l}$ of M-280 streptavidin dynabeads (Thermo Scientific, USA) over night at $4^{\circ} \mathrm{C}$. After washing with the beads for six times with DEPC-PBS buffer. The RNAprotein complex was boiled in $1 \times \mathrm{SDS}$ buffer for $5 \mathrm{~min}$. The retrieved protein was detected using standard western blotting techniques and silver staining.

For mass spectrometry process, in brief, which include protein digestion, MS, database retrieval and protein identification. Firstly, protein digestion, each sample was allowed to proceed at $56^{\circ} \mathrm{C}$ for $1 \mathrm{~h}$ in $10 \mathrm{Mm}$ dithiotreitol and add $55 \mathrm{mM}$ iodoacetamide incubated in the dark for $45 \mathrm{~min}$ at room temperature. The gel pieces were washed with $100 \mu \mathrm{l}$ of $25 \mathrm{~mm}$ $\mathrm{NH} 4 \mathrm{HCO} 3$ for $10 \mathrm{~min}$ and dehydrated with $100 \mu \mathrm{l}$ of $25 \mathrm{~mm}$ $\mathrm{NH} 4 \mathrm{HCO} 3$ in $50 \%$ acetonitrile for $5 \mathrm{~min}$ for two times. Following drying in a SpeedVac, the gel pieces were mixed with $12.5 \mathrm{ng} / \mu \mathrm{l}$ of trypsin and incubated on ice for $40 \mathrm{~min}$ and $25 \mathrm{~mm} \mathrm{NH} 4 \mathrm{HCO} 3$ was added as needed to cover the gel pieces. Digestion was then carried out at $37^{\circ} \mathrm{C}$ overnight. Using $60 \%$ acetonitrile, $0.2 \%$ TFA to extract the tryptic peptides from the gel pieces. Following $20 \mathrm{~min}$ of vortex and $5 \mathrm{~min}$ of sonication, the supernatant was taken and saved. Following the evaporation of acetonitrile in a SpeedVac, the sample was desalted with a C18 ZipTip (Millipore), and half of the eluate was analyzed with nanoLC-MS/MS. Secondly, the samples were resuspended with $30 \mu$ l solvent $\mathrm{C}$ respectively (C: water with $0.1 \%$ formic acid), separated by nanoLC and analyzed by on-line electrospray tandem mass spectrometry. $10 \mu$ peptide sample was loaded onto the trap column (Thermo Scientific Acclaim PepMap C18, $100 \mu \mathrm{m} \times 2 \mathrm{~cm}$ ), with a flow of $10 \mu \mathrm{l} / \mathrm{min}$ for $3 \mathrm{~min}$ and subsequently separated on the analytical column (Acclaim PepMap C18, $75 \mu \mathrm{m} \times 15 \mathrm{~cm}$ ) 
with a 90-min linear gradient, from 5\% D (D: ACN with $0.1 \%$ formic acid) to $55 \% \mathrm{D}$. The column was re-equilibrated at initial conditions for $10 \mathrm{~min}$. The column flow rate was maintained at 300 $\mathrm{nl} / \mathrm{min}$. The electrospray voltage of $2 \mathrm{kV}$ versus the inlet of the mass spectrometer was used. Thirdly, tandem mass spectra were processed by PEAKS Studio version 8.5 (Bioinformatics Solutions Inc., Waterloo, Canada). PEAKS DB was set up to search the UniProt-homo sapiens database (version 201712, 72029 entries) assuming trypsin as the digestion enzyme. Peptides were filter by $1 \%$ FDR and 1 unique peptide.

\section{RNA Immunoprecipitation (RIP) Assay}

RIP was performed using the EZ-Magna RIP kit (Millipore, Billerica, MA) following the manufacturer's protocol. 231 cells at 80-90\% confluency was scraped off the tissue culture plate, then lysed in complete RIP lysis buffer. A total of $200 \mu \mathrm{l}$ of whole cell extract was incubated with RIP buffer containing magnetic beads conjugated with antibodies against PABPC4 and TAB3 or control IgG (Millipore) overnight at $4^{\circ} \mathrm{C}$. The beads were washed with wash buffer, then the complexes were incubated with $0.1 \%$ SDS and $0.5 \mathrm{mg} / \mathrm{ml}$ Proteinase $\mathrm{K}\left(30 \mathrm{~min}\right.$ at $\left.55^{\circ} \mathrm{C}\right)$ to remove proteins. The RNA concentration was measured using a NanoDrop spectrophotometer (Thermo Scientific, USA) and its quality was assessed using a bioanalyzer (Agilent, Santa Clara, CA). Finally, immunoprecipitated RNA was purified and analyzed by qRT-PCR.

\section{Subcellular Fractionation}

The separation of nuclear and cytosolic fractions was performed using the NE-PER Nuclear and Cytoplasmic Extraction Reagents Kit (Thermo, USA) following the manufacturer's instructions. In brief, for adherent cells, harvest with trypsin-EDTA and then centrifuge at $500 \times \mathrm{g}$ for $5 \mathrm{~min}$; Wash cells by suspending the cell pellet with PBS and centrifuge at $500 \times \mathrm{g}$ for $2 \mathrm{~min}$ for two times; Add ice-cold $200 \mu \mathrm{l}$ CER I to the cell pellet, and vortex the tube vigorously on the highest setting for $15 \mathrm{~s}$ to fully suspend the cell pellet. Incubate the tube on ice for $10 \mathrm{~min}$, add ice-cold $11 \mu \mathrm{l}$ CER II, vortex the tube for $5 \mathrm{~s}$ on the highest setting. Incubate tube on ice for $1 \mathrm{~min}$; Centrifuge the tube for $5 \mathrm{~min}$ at $16,000 \mathrm{~g}$, transfer the supernatant (cytoplasmic extract) to a tube; suspend the pellet with $200 \mu \mathrm{l}$ ice-cold NER, vortex on the highest setting for $15 \mathrm{~s}$ every $10 \mathrm{~min}$, for a total of $40 \mathrm{~min}$; Centrifuge the tube at $16,000 \times \mathrm{g}$ for $10 \mathrm{~min}$; Immediately transfer the supernatant (nuclear extract) fraction to a clean pre-chilled tube, store extracts at $-80^{\circ} \mathrm{C}$ until use. RNA isolation using TRIzol reagent (Invitrogen) and using PrimeScript RT Reagent Kit (Takara, Dalian, China) for cDNA production. $\beta$-Actin was used as the cytoplasmic endogenous control. U6 small nuclear RNA was used as the nuclear endogenous control for qPCR.

\section{RACE Assay}

We used a SMARTer RACE cDNA Amplification kit (Clontech, California, USA) to determine the transcriptional initiation and termination sites of Lnc-PCIR, according to manufacturer's instructions. The sequences for the gene-specific PCR primers used for 5' and 3' RACE analysis was given in Supplementary Table 2.

\section{RNA Scope Assay}

RNA in situ hybridization was performed using RNAScope ${ }^{\circledR}$ Multiplex Reagent Kit for Tissues (ACD, Life technologies, USA) to analysis the RNA level of Lnc-PCIR in human TNBC tissues and adjacent normal tissues. In brief, deparaffinized tissue sections were hybridized with the Lnc-PCIR probe and negative control probe at $40^{\circ} \mathrm{C}$ for $2 \mathrm{~h}$. After hybridizations, sections were subjected to signal amplification, Gill's hematoxylin counterstaining, and scanning (Aperio ScanScope CS, Leica Biosystems, Nussloch, Germany) at $40 \times$ magnification. Fast Red semiquantitative image analysis was performed using the Aperio RNA ISH algorithm, which automatically quantifies the staining across whole slides and counts individual molecular signals and clusters in the cells. The obtained results are divided into three ranges: 1 , which includes cells containing two to five dots per cell; 2, which includes cells containing 6-20 dots per cell; and 3 , which includes cells containing more than 20 dots per cell.

\section{Tumor Xenograft Experiments}

Female NOD/SCID nude mice (4 weeks old) were obtained from the Shanghai Model Organisms Center (Shanghai, China). And housed and maintained in laminar airflow cabinets under specific pathogen-free conditions. Subsequently, the stable lenti-P-LncPCIR or control 231 cells $\left(1 \times 10^{7}\right.$ cells/mice in $200 \mu$ sterile PBS $)$ were injected subcutaneously into NOD/SCID nude mice. Tumor growth was measured after 1 week, and tumor volumes were calculated by the formula: volume $\left(\mathrm{cm}^{3}\right)=\left(\right.$ length $\times$ width $\left.{ }^{2}\right) / 2$. After 4 weeks, the mice were sacrificed and the tumors were collected and weighed. For in vivo metastasis assay, $1 \times 10^{6}$ lentiP-Lnc-PCIR or control 231 cells (in $50 \mu$ of sterile PBS) were orthotopically injected directly into the inguinal mammary fat pads of mice in ( $n=10$ in each group). All procedures were conducted in accordance with the Guidelines for the Care and Use of Laboratory Animals with the approval of the Ethics Committee of the Fujian Medical University.

\section{Statistical Analysis}

All experiments were performed in triplicate. Statistical analyses were performed using SPSS (version 23.0, SPSS Inc.) or GraphPad Prism software (version 7.0, USA). Clinicopathological characteristics were analyzed by chi-square tests. Survival curves were generated using the Kaplan-Meier method and log-rank tests. Univariate and multivariate Cox regression analyses were conducted to identify the independent factors. Student's t-test or the Mann-Whitney U test was used for comparison between two groups depending on distribution. P (two-sided) less than 0.05 was considered to indicate statistical significance. All data were presented as the mean \pm standard error of the mean (SEM).

\section{RESULTS}

\section{Identification of Clinically Relevant IncRNAs Overexpressed in TNBC}

In order to identify lncRNAs that play a role in TNBC, we used RNA-sequencing (RNA-seq) data from 1084 patients available in 
the TCGA database (The Cancer Genome Atlas). We classified the tumors with available PAM50 (Prediction Analysis of Microarray 50) molecular subtype annotation (25), obtaining a final cohort of 110 TNBC patients (cohort 1) (26). Using differentially expressed gene analysis, we identified a subset of $\operatorname{lncRNAs}$ overexpressed with clinically relevant in the TNBC subtype (fold change $>4$ ) compared to normal tissue (Figures 1A, B and Supplementary Excel 1). Subsequently, the top four upregulated lncRNAs are selected: RP11214F16.8 (Lnc-PCIR), LOC645249, SNHG3, and LINC00160. The RNA level of Lnc-PCIR was confirmed in 550 paired TNBC tissues and paired tumor-adjacent non-tumor tissues (Cohort2) (Figure 1C). The univariate Cox proportional hazards regression method revealed that Lnc-PCIR showed the significantly prognostic value in TNBC tissue (Figure 1C). So, we choose the Lnc-PCIR for further study. Moreover, the RNA levels of Lnc-PCIR were confirmed by quantitative real-time polymerase chain reaction (q-PCR) analysis in 110 paired TNBC tissues and paired tumor-adjacent non-tumor tissues (Cohort3). Compared with matched normal tissues, LncPCIR was significantly up-regulated in TNBC tissues (Figure 1D). We also employed the RNA Scope assay to analyze Lnc-PCIR RNA
A

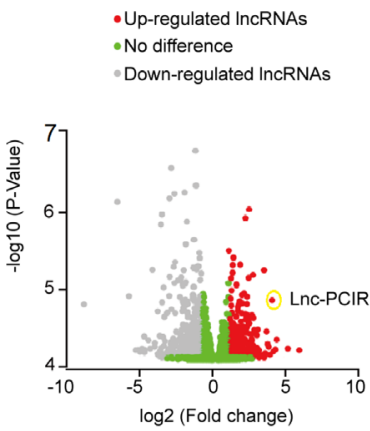

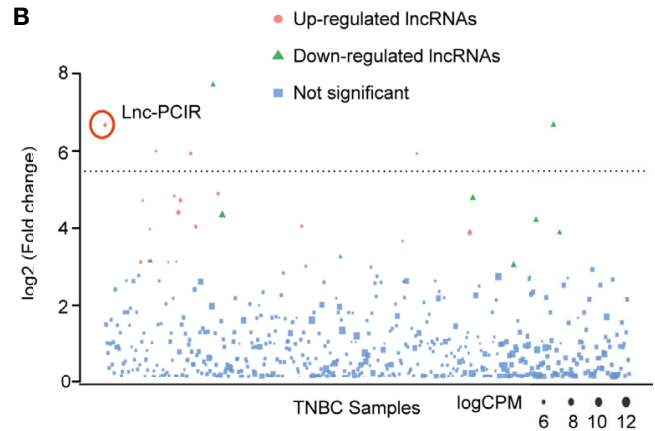

C
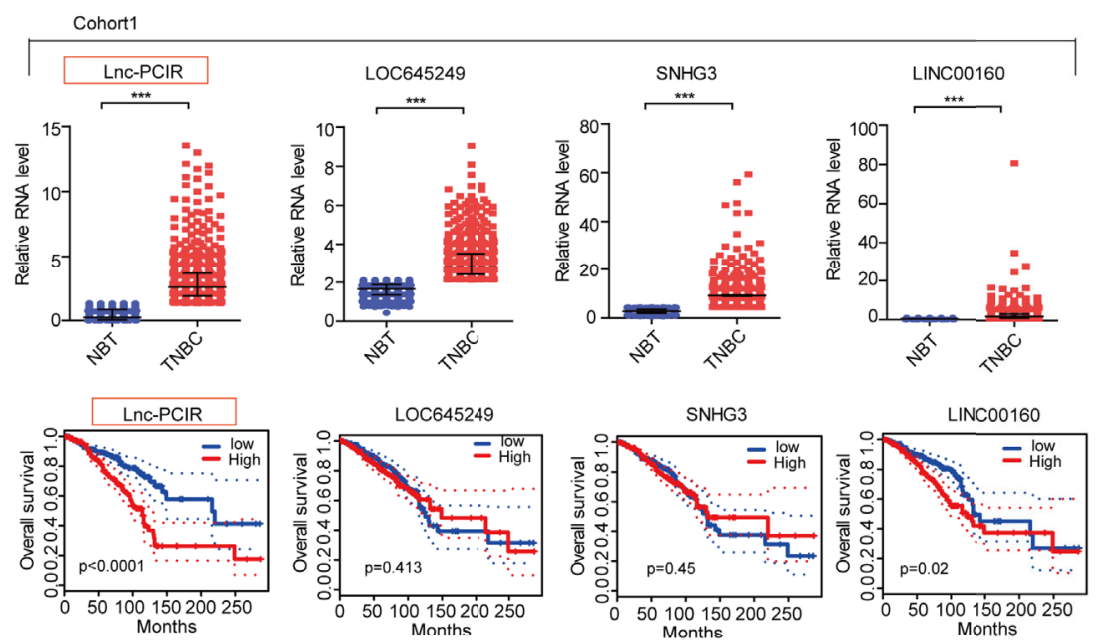

D
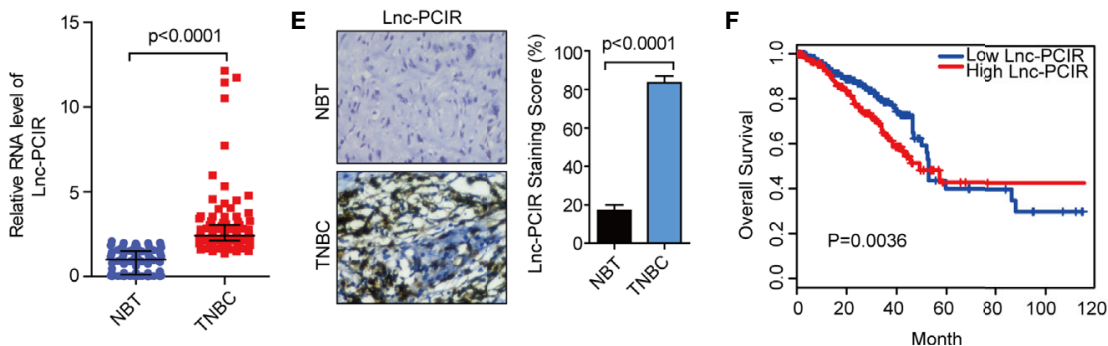

FIGURE 1 | Lnc-PCIR upregulated and predicted the worse survival in triple negative breast cancer. (A, B) Volcano plot and Manhattan plot analysis of RNA-

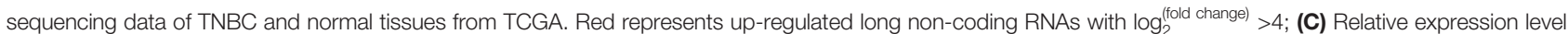
and overall survival of screened IncRNAs in TCGA data of TNBC samples; (D) qRT-PCR results showed that Lnc-PCIR expression was significantly upregulated in 110 pairs of TNBC tissues and non-tumor tissues (NTs); (E) RNA Scope assay to detect the Lnc-PCIR RNA level in TNBC and adjacent normal tissues. Left panel: representative images; right panel: statistical analysis of the staining; (F) Kaplan-Meier survival analysis of Lnc-PCIR expression in TNBC patients ( $\mathrm{n}=110$ ). All $p$-values calculated by independent sample t-test ${ }^{* * *}$ significant values of $\left.<0.001\right)$. 
level, results confirmed Lnc-PCIR has significantly higher level in TNBC tissues (Figure 1E). Patients with higher levels of the LncPCIR exhibited poor survival outcomes $(P=0.0036)$ (Figure 1F). These data together indicated that Lnc-PCIR was significantly upregulated and related to the overall survival in TNBC tissues, and might be involved in the progression of TNBC.

\section{Overexpressed Lnc-PCIR Promoted Cell Migration, Invasion, and Proliferation In Vitro and In Vivo}

We first analyzed the basic characteristic of Lnc-PCIR in breast cancer cells. Lnc-PCIR (ENSG00000280710.2) located on 13 q32.3 and has only one transcript with three exons (Supplementary Figure 1A). Lnc-PCIR is widely expressed in different breast cancer cell lines, and the RNA level was significantly up-regulated in TNBC cell lines (Supplementary Figure 1B). Next, we examined the subcellular localization of Lnc-PCIR, finding that Lnc-PCIR predominately resides in the nucleus in 231 and BT549 cells by qRT-PCR (Supplementary Figure 1C). By Coding Potential Calculator (27) (http://cpc.cbi. pku.edu.cn/) and the PhyloCSF codon substitution frequency analysis (28), Lnc-PCIR has low protein-coding ability (Supplementary Figures 1D, E). Moreover, we performed the RACE assay (rapid amplification of cDNA ends) and Northern blot assay to confirm the Lnc-PCIR is a 987-bplong intergenic non-protein-coding RNA in breast cancer cells (Supplementary Figures 1F, G).

To assess the effect of Lnc-PCIR on TNBC cell migration and invasion, we performed transwell assay and wound-healing assay. Two independent small interfering RNAs (siRNAs) for Lnc-PCIR significantly decreased the migration and invasion of the 231 and BT549 cells (Figures 2A-D and Supplementary Figure 2A), and vice-versa, Lnc-PCIR overexpression by a lentivirus vector ( $\mathrm{pCDH}$ - Lnc-PCIR, shorted in P-Lnc-PCIR) promoted 231 and BT549 cells the migration and invasion of the 231 and BT549 cells (Figures 2E-G and Supplementary Figure 2B). On the other hand, Lnc-PCIR knockdown significantly decreased 231 and BT549 cells growth and colony formation (Figures 3A-C), whereas, Lnc-PCIR induction increased cell growth and colony formation (Figures 3D-F).

To further explore the growth-promoting effects of Lnc-PCIR on TNBC cells in vivo, we evaluated the promoting effects of LncPCIR on cell metastasis. The stable P-Lnc-PCIR 231 cells were transplanted into the fat pad of nude mice. The metastatic nodules in the lung were significantly increased in the P-LncPCIR group (Figure 4A). Hematoxylin-eosin staining showed that the metastatic foci derived from the P-Lnc-PCIR cells dramatically increased in the lung (Figure 4B). We also subcutaneously injected stable P-Lnc-PCIR 231 cells into nude mice. Both the volumes and weights of the tumors in the P-LncPCIR group were markedly higher than those in the control group (Figures 4C-E) with no significant change in body weight of nude mice (Figure 4F), demonstrating that P-Lnc-PCIR promotes the tumorigenicity of the TNBC cells in vivo. Taken together, these findings suggest that Lnc-PCIR acts as an oncogenic driver in the development and progression of TNBC.

\section{Identified the Lnc-PCIR Binding Proteins}

To explore the molecular mechanism underlying the oncogenic activity of Lnc-PCIR in TNBC progression, we performed RNA pull-down assays to identify the proteins associated with LncPCIR in the 231 cells. The results from three independent LncPCIR pull-down experiments repeatedly showed specific bands at approximately $70 \mathrm{KD}$ and $90 \mathrm{KD}$ via mass spectrometry (Figure 5A and Supplementary Table 4). Nine potential interacting proteins were obtained based on unique peptide number $>5$ and peptide number $>10$ in the three independent experiments and were absent in the corresponding antisense groups (Figure 5A). After confirming in two independent experiments, we observed that sense but not antisense LncPCIR, was specifically associated with TGF-beta activated kinase 1 (MAP3K7) binding protein 3 (TAB3) and Poly(A) binding protein cytoplasmic 4 (PABPC4) (Figure 5B). Moreover, RIP assays showed that the antibodies of TAB3 or PABPC4 could significantly enrich Lnc-PCIR (Figure 5C), whereas the GAPDH antibody and IgG as the negative control. In addition, unbiased transcriptome profiling was performed using RNA-sequencing in 231 cells transfected with two independent si-Lnc-PCIR to investigate the related signaling pathways and biological process. GSEA (Gene Set Enrichment Analysis) and GO analysis (Gene Ontology Analysis) showed that top four signaling pathways Lnc-PCIR involved in which include: TNFA signaling via NFKB pathway, Hypoxia, Epithelial-mesenchymal Transition (EMT) and Estrogen response early in 231 cells (Figure 5D and Supplementary Excel 2). Moreover, we verified the top-scoring genes altered in TNFA signaling via NFKB pathway and confirmed that LncPCIR dramatically affected the genes expression level that are highly associated with tumorigenesis (Figure 5E). Taken together, these findings demonstrated that Lnc-PCIR is an oncogenic driver through activating TNF- $\alpha / N F-\kappa B$ signaling pathway by binding with TAB3 and PABPC4 in TNBC and the molecular mechanisms between them need further explored.

\section{Lnc-PCIR Enhances the Protein Levels of TAB3 and PABPC4}

In order to explore the regulatory mechanism among Lnc-PCIR, TAB3 and PABPC4, we first analysis the binding fragment/ domain between them, a series of deletion was constructed based on the secondary structure of Lnc-PCIR (http://www.Incipedia. org/, Figure 6A). The 201-507 nt (\#2) fragment of Lnc-PCIR mediates the interaction with TAB3, while the 625-987 nt (\#3) fragment of Lnc-PCIR is required and sufficient for the association with PABPC4. Additionally, we also construct the FLAG-tagged full-length and truncated TAB3 or PABPC4. RIP assays showed CUE domain (1-244 aa) of TAB3 was the vital domain mediate the interaction with Lnc-PCIR (Figure 6B), and PABP-1234 super family domain (11-624 aa) of PABPC4 is required for its association with Lnc-PCIR (Figure 6C). Next, we sought to determine the functional relevance of the association between Lnc-PCIR and TAB3/PABPC4. Knockdown of LncPCIR significantly decreased the mRNA level of TAB3, and vice versa (Figure 6D). But knockdown and overexpressed Lnc-PCIR 
A

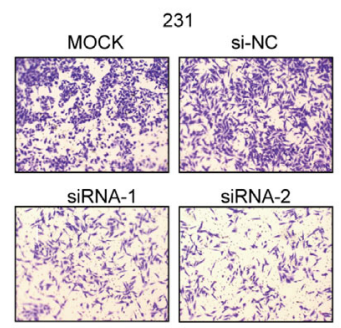

C

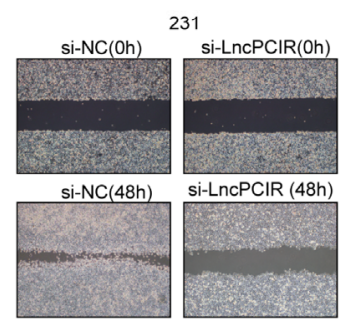

E

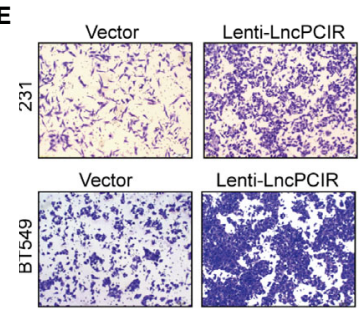

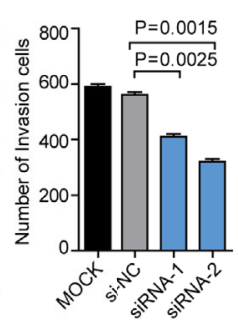

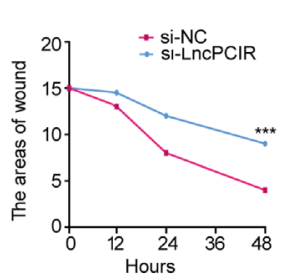

D
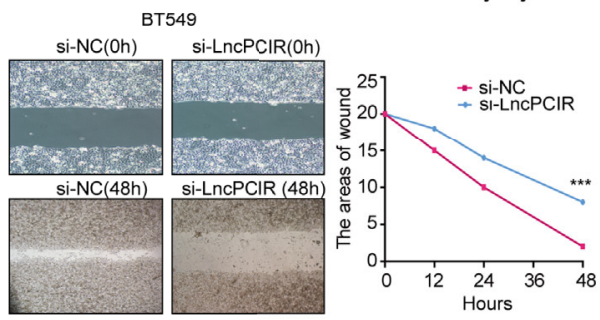

231

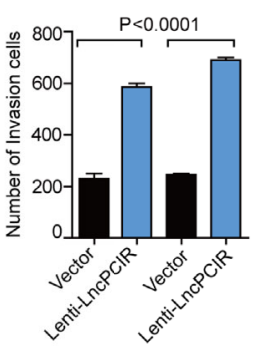

$\mathbf{F}$
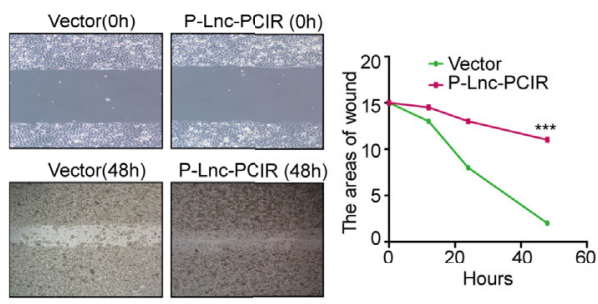

G

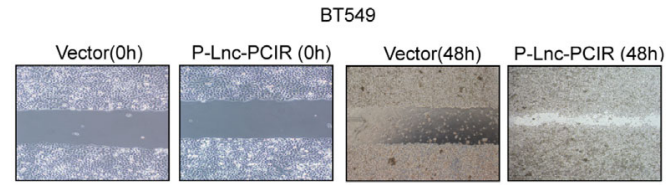

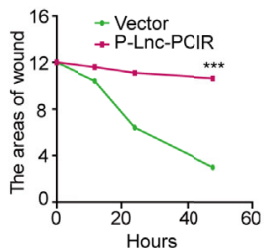

FIGURE 2 | Lnc-PCIR increases TNBC cell invasion and migration in vitro. (A, B) Transwell invasion assays in the 231 (A) and BT549 (A) cells with Lnc-PCIR knockdown; (C, D) Wound-healing assays in the 231 (A) and BT549 (A) cells with Lnc-PCIR knockdown; (E) Transwell invasion assays in the 231 (A) and BT549 (A) cells with Lnc-PCIR overexpressed; (F, G) Wound-healing assays in the 231 (A) and BT549 (A) cells with Lnc-PCIR overexpressed. All p-values calculated by independent sample t-test ( ${ }^{\star \star *}$ significant values of $\left.<0.001\right)$.

has non-effect of mRNA level of PABPC4 (Figure 6D). Western blot assay revealed overexpressed Lnc-PCIR increased the expression level of TAB3 and PABPC4, knockdown of LncPCIR could significantly reduce the protein level of TAB3/PABPC4 in 231 cells (Figure 6E). Moreover, actinomycin D, which effectively inhibits the de novo synthesis of RNA, was used to explore the stability of TAB3 regulated by Lnc-PCIR. Overexpression of Lnc-PCIR could increase the half-life and steady-state level of TAB3, whereas the depletion of Lnc-PCIR resulted in a decreased half-life and RNA level of TAB3 (Figure 6F), revealing that Lnc-PCIR specifically regulate the stability of TAB3 in the TNBC cells. Meanwhile, we found PABPC4 could specifically bind with TAB3 in 231 cells (Figures 6G, H). PABPC4, a Poly (A)binding protein, is expressed at a higher level in colon cancer and lung adenocarcinoma compared to normal tissues $(29,30)$. However, the expression profile and role of PABPC4 in TNBC remains unknown. Given this, we speculate whether PABPC4 can directly bind to the TAB3 mRNA. RNA pulldown assay was conducted with biotinylated TAB3 mRNA, results showed overexpressed Lnc-PCIR strengthened the binding of PABPC4 and mRNA of TAB3 (Figure 6I). Both PABPC4 and TAB3 are overexpressed in TNBC tissues, and a strong correlation between them (Supplementary Figures 3A-C). Furthermore, knockdown of PABPC4 or TAB3 reduced the cell growth and invasion abilities of the 231 cells (Supplementary Figures 3D, E and Supplementary Figures 4A-D). These findings suggest higher RNA level of LncPCIR could strengthening the stability of TBA3 mRNA, by enhancing the binding state of PABPC4 to TAB3 in TNBC cells. 
A

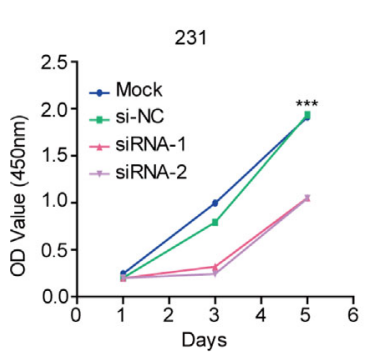

D

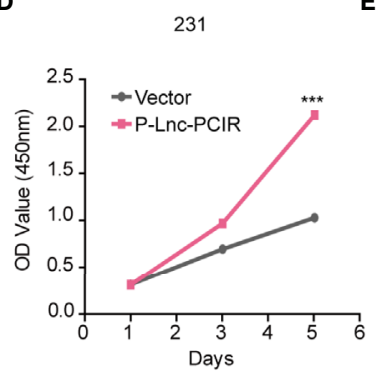

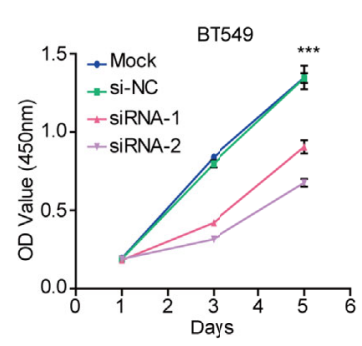

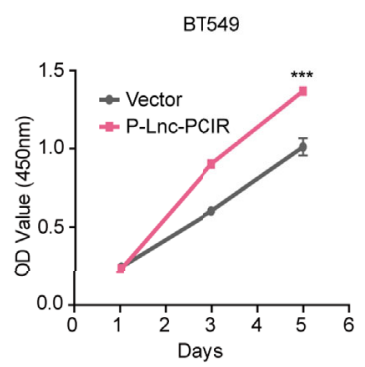

C
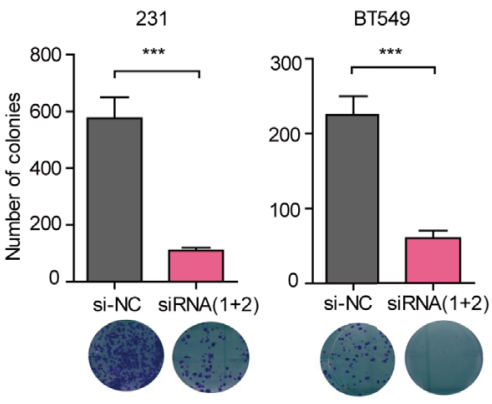

$\mathbf{F}$

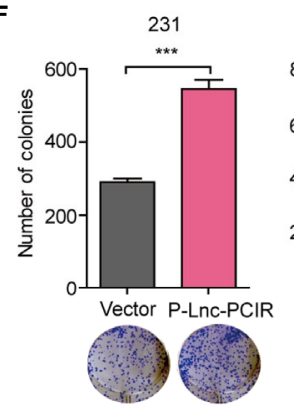

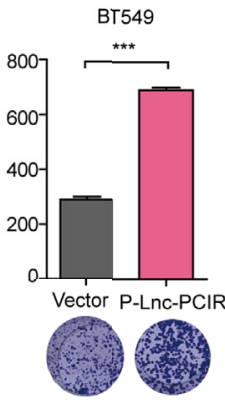

FIGURE 3 | Lnc-PCIR increases TNBC cell proliferation and colony formation in vitro. (A-C). CCK-8 assays (A, B) and Colony formation assays (C) in the 231 and BT549 cells with knockdown of Lnc-PCIR; (D-F). CCK-8 assays (D, E) and Colony formation assays (F) in the 231 and BT549 cells with overexpression of Lnc-PCIR. All $p$-values calculated by independent sample t-test ( ${ }^{\star \star *}$ significant values of $\left.<0.001\right)$.

\section{Lnc-PCIR Blocks PABPC4 Proteasome- Dependent Ubiquitination Degradation}

Although Lnc-PCIR had no significant effect on the mRNA level of PABPC4 (Figure 6D), the protein levels of PABPC4 were dramatically increased when overexpressing Lnc-PCIR and were reduced when silencing Lnc-PCIR in TNBC cells (Figure 6E). To explore the mechanisms underlying this phenomenon, we wonder whether it affects the stability of the PABPC4 protein level. Thus, following treatment with a protein-synthesis inhibitor cycloheximide (CHX), Lnc-PCIR knockdown accelerate the PABPC4 degradation, whereas Lnc-PCIR activation increased the half-life of the PABPC4 protein in the TNBC cells (Figure 7A). Besides, we also treated cells with a proteasome inhibitor MG132, the accumulation of endogenous PABPC4 in cells overexpressing Lnc-PCIR was remarkable greater (Figure 7B), indicating that higher level Lnc-PCIR might inhibit the proteasome-dependent degradation of PABPC4 in TNBC cells. Furthermore, the ubiquitination levels of PABPC4 significantly increased in the sh-Lnc-PCIR cells, whereas the ubiquitination levels of PABPC4 sharply decreased in the overexpressed LncPCIR cells (Figure 7C). Collectively, these results indicated that Lnc-PCIR can increase the stability of PABPC4 through blocking its ubiquitin/proteasome-dependent degradation.

\section{TAB3 as the Functional Downstream Mediator of Lnc-PCIR to Activating TNF- $\alpha /$ NF- $\kappa B$ Pathway}

Previous studies have shown that TAB2 which tightly associated with TAB3 is involved in NF- $\kappa \mathrm{B}$ activation (31). Considering the effect of Lnc-PCIR on TAB3 protein level, we hypothesize that Lnc-PCIR may exert its biological effects through regulating the association of TAB3 and TAB2. The Co-IP study showed that endogenous $\mathrm{TAB} 2$, were detected in the immunoprecipitant with TAB3 antibody, and the association of TAB2 and TAB3 was remarkable decreased due to Lnc-PCIR overexpression (Figure 7D). Furthermore, functional recovery assay showed deleting TAB3 in the P-Lnc-PCIR cells significantly inhibited the invasion abilities, indicating that $\mathrm{TAB} 3$ is an important downstream effector of P-Lnc-PCIR in TNBC cells (Figure 7E). On the other hand, the activation of NF- $\mathrm{KB}$ signaling by treatment with TNF $\alpha(10 \mathrm{ng} / \mathrm{ml})$ rescued the decreased cell invasion induced by the knockdown of TAB3. We also treated cells with the NF- $\mathrm{KB}$ inhibitor caffeic acid phenethyl ester (CAPE, $10 \mathrm{ng} / \mathrm{ml}$ ), the transwell assay showed that blockade of NF- $\mathrm{KB}$ signaling dramatically decreased Lnc-PCIR induced cell migration and invasion (Figure 7E). To further clarify the mechanism by which Lnc-PCIR regulates via the $\mathrm{NF}-\mathrm{\kappa B}$ signaling in TNBC cells, we measured the changes in phosphorylated $I \kappa B \alpha(p-I \kappa B \alpha)$, phosphorylated-p65 (p-p65) and total p65 expression in Lnc-PCIR knockdown or overexpression 231 cells. The results showed that the knockdown of Lnc-PCIR can significantly decrease $\mathrm{p}-\mathrm{I} \kappa \mathrm{B}$ and p-p65 in 231 cells and vice-versa (Figure 7F). TNBC patients in the high TAB3 expression group had a much shorter median survival time than those in the low TAB3 expression group (Figure 7G). And TAB3 showed a positive correlation with Lnc-PCIR in TNBC tissues (Figure $\mathbf{7 H}$ ). Taken together, Lnc$\mathrm{PCIR}$ as the oncogenic driver in TNBC, they make a meaningful 
A

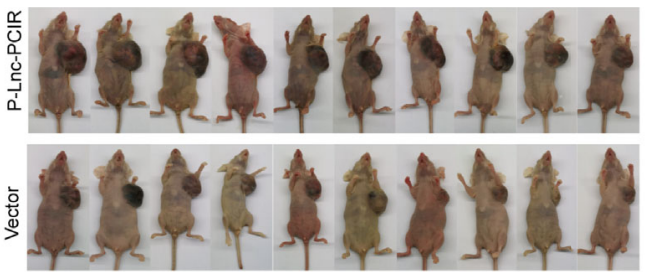

\begin{tabular}{lccc}
\multicolumn{4}{c}{ Analysis of In Vivo events } \\
\hline & P-Lnc-PCIR & Vector & p value \\
\hline nude mice tumorigenesis & $10 / 10$ & $10 / 10$ & $\mathrm{~ns}$ \\
Lung metastasis & $10 / 10$ & $4 / 10$ & $<0.0001$ \\
\hline
\end{tabular}

B
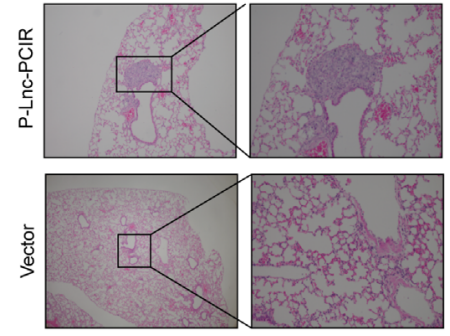

D

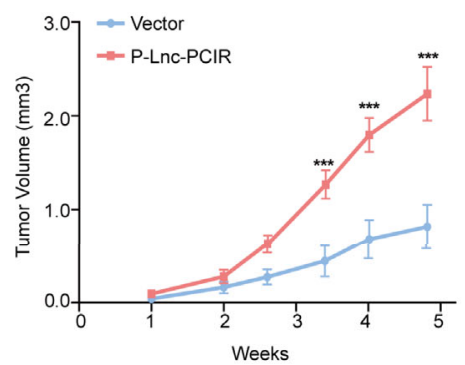

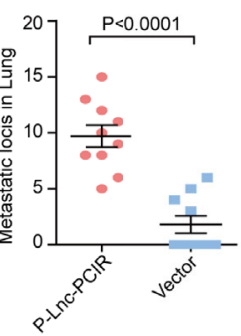

E

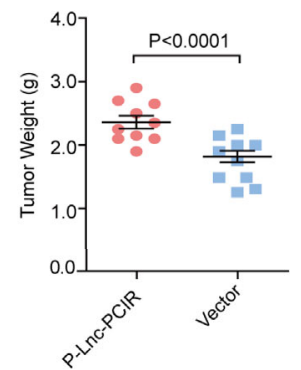

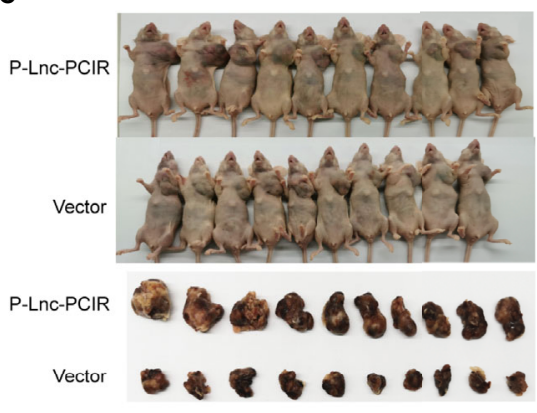

$\mathbf{F}$

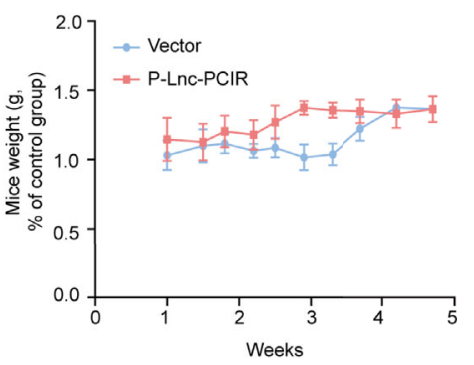

FIGURE 4 | Lnc-PCIR increases TNBC cell metastasis and cell growth in vivo.(A, B) Statistics analysis of the metastatic foci in the lung obtained from nude mice injection with the stable P-Lnc-PCIR 231 cells detected by hematoxylin-eosin staining; (C-E). Representative image of nude mouse models bearing subcutaneous tumor xenografts (C); Tumor volumes (D) and tumor weights (E) were measured in the P-Lnc-PCIR 231 cells and negative-control groups in the xenograft mouse models; (F) The weight of nude mice in the P-Lnc-PCIR 231 cells group and negative-control group. All $p$-values calculated by independent sample t-test $\left({ }^{* \star *}\right.$ significant values of $\left.<0.001\right)$.

contribution to tumor progression through activating the NF- $\mathrm{KB}$ signaling by associated with TAB3.

\section{DISCUSSION}

Long non-coding RNAs were found to be deregulated in a variety of diseases, especially cancer (32). Understanding the precise molecular mechanism by which lncRNAs function is vital for exploring new potential strategies for early diagnosis and therapy. In this study, from RNA-sequencing data, we identified LncPCIR, is a clinically relevant lncRNA displays a remarkable trend of increased expression in TNBC tissue. Importantly, higher LncPCIR levels predicted lower overall survival rates in TNBC patients, supporting that Lnc-PCIR may be a promising prognostic biomarker for TNBC. Lnc-PCIR showed the strong oncogenic activity by promoting TNBC cell tumor invasion and metastasis, proliferation, tumorigenicity in vitro and in vivo. And these results may suggest Lnc-PCIR involved in cancer-related biological processes and pathways which lead to TNBC tumorigenesis and progression.

Through the study on the mechanisms driven by Lnc-PCIR, we performed RNA Pulldown and RNA-sequencing assay, and found Lnc-PCIR could directly binds to TAB3 and PABPC4. TAB3 bind to the $5^{\prime}$ terminus of Lnc-PCIR (210-624 nt), whereas the 3' terminus of Lnc-PCIR (625-987 nt) accounts for its association with PABPC4; therefore, it was not surprising that these two proteins could bind to each other.

TABs family which include TAB1, TAB2 and TAB3 have been identified as the specific binding partner proteins of TAK1 
A

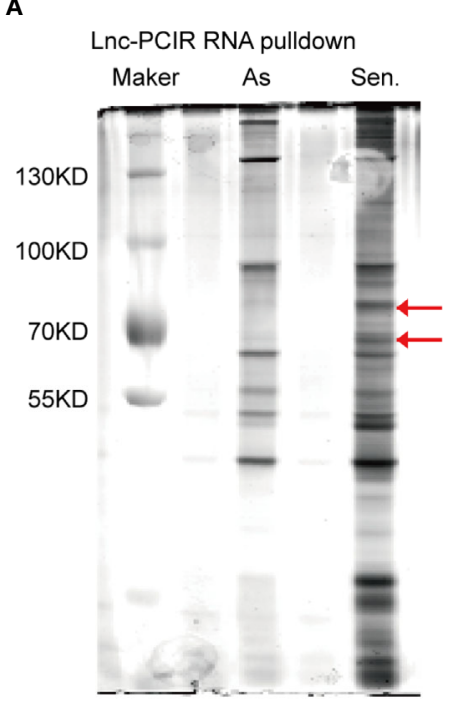

Lnc-PCIR RNA Pulldown-Mass

\begin{tabular}{|l|c|}
\hline $\begin{array}{l}\text { Bead only } \\
\text { Collagen type I alpha 2 isoform 1 }\end{array}$ & $1 / 1$ \\
Collagen alpha-1(III) chain & $1 / 1$ \\
\cline { 1 - 1 } Antisense & \\
Myosin-9 & $1 / 1$ \\
Myosin-10 & $1 / 1$ \\
\cline { 1 - 1 } Sense & \\
TGF-Beta-Activated Kinase 1-Binding Protein 3 & $15 / 15$ \\
TGF-Beta-Activated Kinase 1-Binding Protein 2 & $10 / 14$ \\
IkB Kinase-Associated Protein 1 & $10 / 14$ \\
TNF Receptor-Associated Factor 6 & $10 / 11$ \\
Interferon alpha 1 & $9 / 10$ \\
Eukaryotic translation initiation factor 5B & $6 / 10$ \\
Poly(A)-Binding Protein, Cytoplasmic 4 & $6 / 10$ \\
RecQ Protein-Like 1 & $5 / 10$ \\
Tol like receptor 4 & $5 / 10$ \\
\hline
\end{tabular}

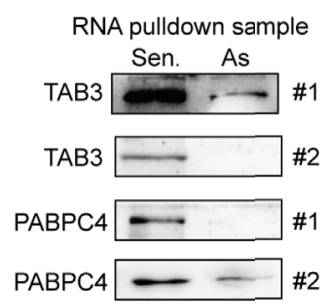

C

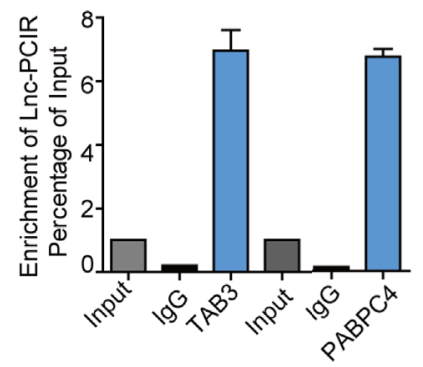

D

Interferon alpha response Estrogen response late Unfolded protein response Interferon gamma response IL2-STAT5 Signaling TNFA signaling via NFKB KRAS signaling Inflammatory response. \begin{tabular}{r|} 
Hypoxia \\
\hline Estrogen response early \\
\hline EMT \\
\hline Apoptosis
\end{tabular}

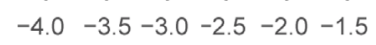
$\log 10$ ( $P$ value $)$
E

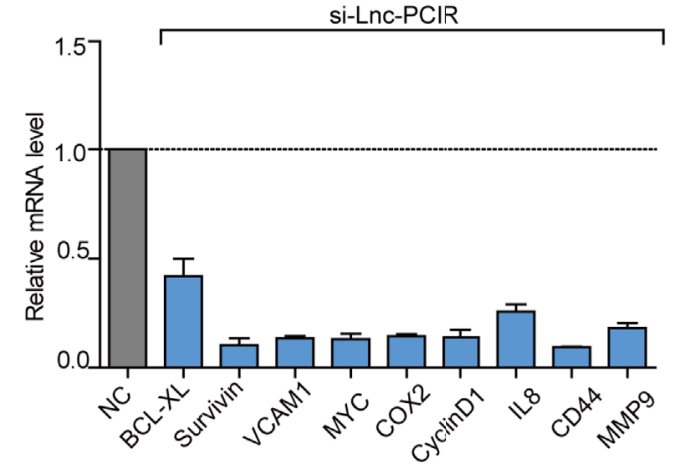

TNFA signaling via NFKB

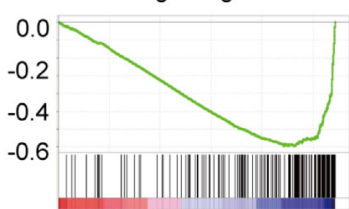

Hypoxia

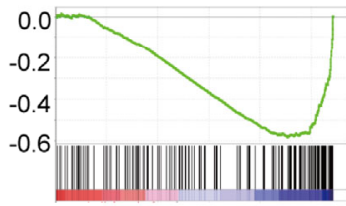

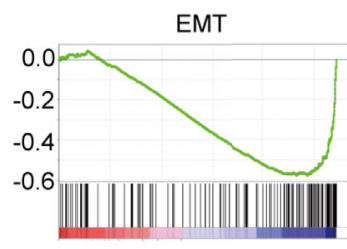

Estrogen response early

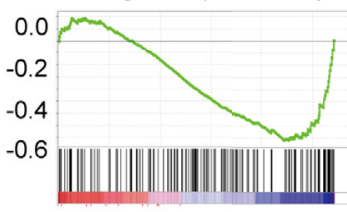

SIZE

- 100

- 125

- 150

-1.5
-2.0
-2.5
-3.0
-3.5
-4.0

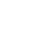

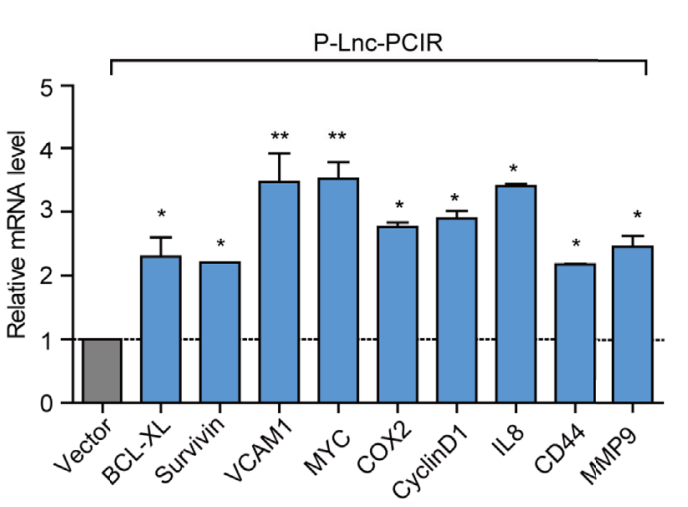

FIGURE 5 | Lnc-PCIR specific directly binding with TAB3 and PABPC4 in 231 cells. (A) Silver staining of SDS-PAGE gel of biotinylated Lnc-PCIR RNA pull-down assays. Red arrows indicated the Lnc-PCIR-Sense-specific bands; (B) Western blot to analysis the interaction partners of Lnc-PCIR using RNA pull-down samples in 231 cell lines; (C) RIP analyses were performed using antibodies against endogenous TAB3 and PABPC4, with IgG as a negative control. The RNA level of the LncPCIR was detected using RT-PCR and normalized to the input; (D) GSEA analysis of RNA-sequencing primary data when knockdown of Lnc-PCIR by two independent siRNAs and representative image of top four signaling pathways showed by GSEA analysis; (E) Top key genes regulated by TNF- $\alpha / N F-\kappa B$ pathway were verified by qRT-PCR when knockdown or overexpressed Lnc-PCIR in TNBC cells. All $p$-values calculated by independent sample t-test ( ${ }^{\star}$ significant values of $<0.05$; ${ }^{* *}$ significant values of $\left.<0.01\right)$. 
A

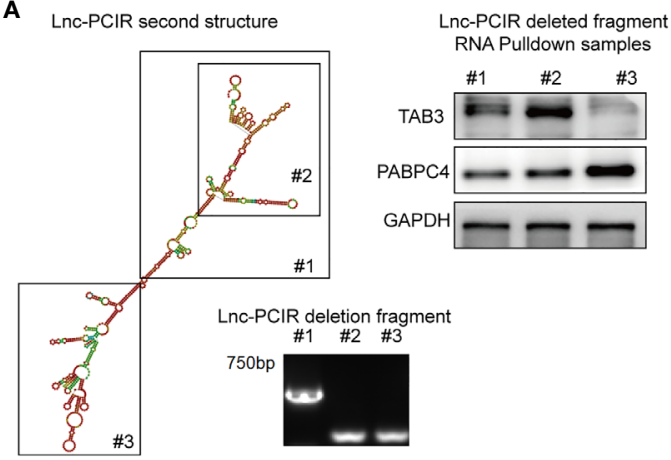

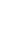

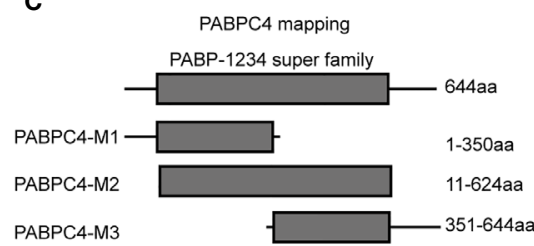

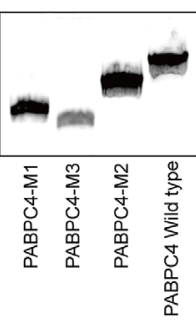
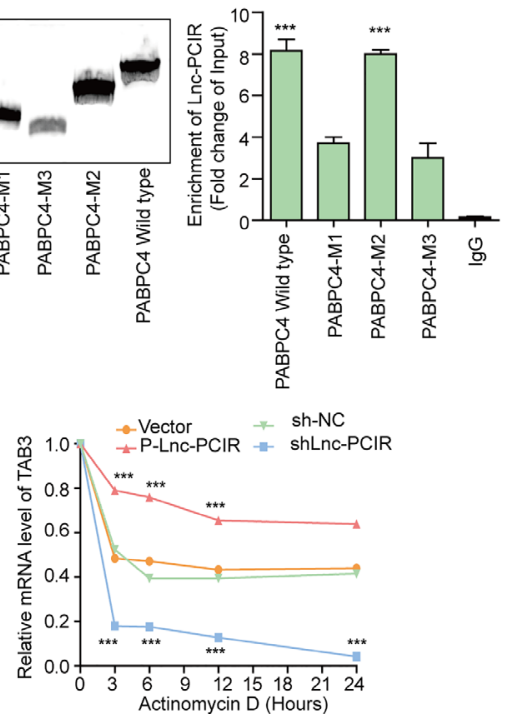

D

E

G

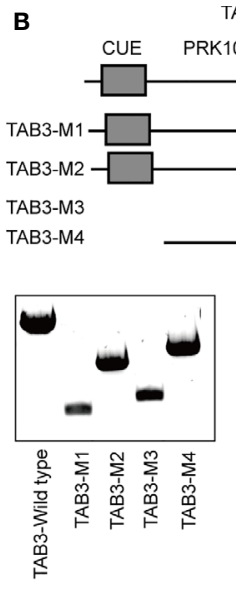

TAB3 mapping

ZNF-RBZ

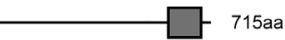

- $246-715$ a
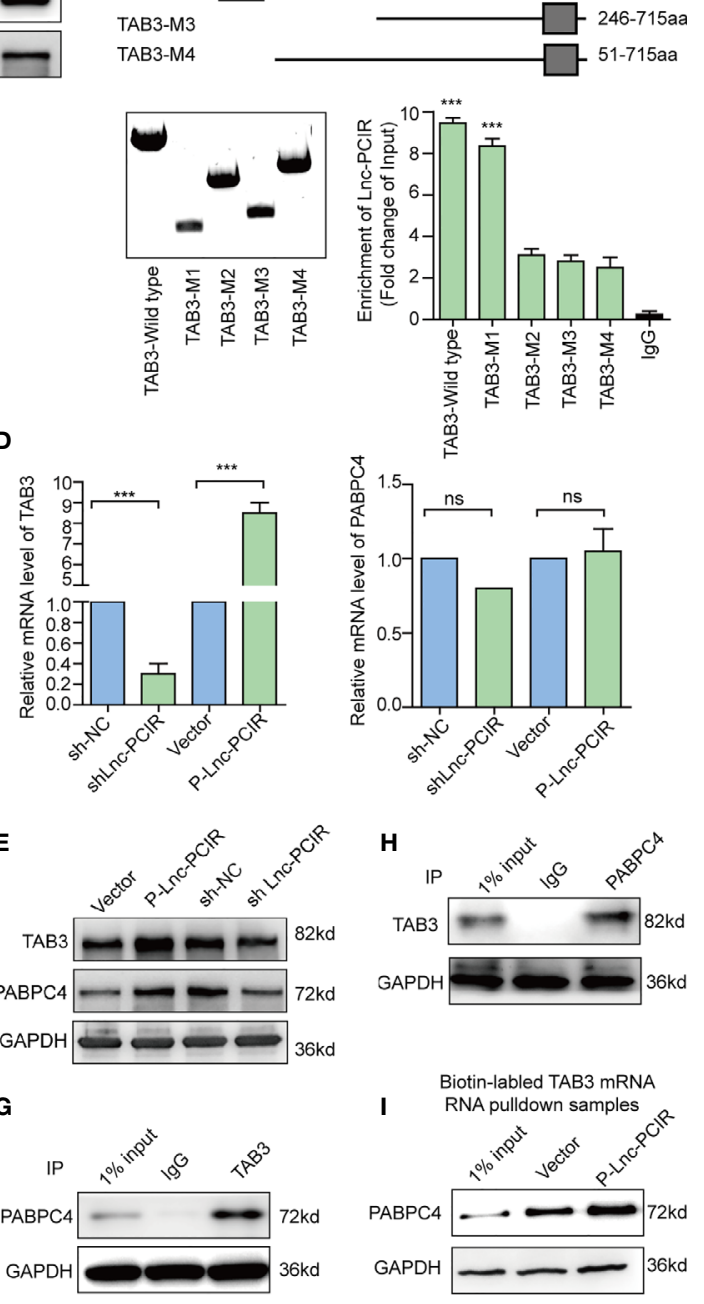

FIGURE 6 | Lnc-PCIR enhances the protein levels of TAB3 and PABPC4. (A) Deletion mapping of the Lnc-PCIR according to the second structure (https:// Incipedia.org/; Western blot of TAB3 or PABPC4 in pull-down samples by full-length biotinylated-Lnc-PCIR or truncated biotinylated- Lnc-PCIR RNA motifs, with GAPDH as the negative control; (B, C) RIP analysis of deletion mapping for the domains of TAB3 (B) or PABPC4 (C) that bind to Lnc-PCIR; (D) The mRNA levels of TAB3 or PABPC4 were quantified by qRT-PCR with Lnc-PCIR knockdown or overexpression; (E) The protein levels of TAB3 or PABPC4 after Lnc-PCIR

overexpression or knockdown. GAPDH served as the internal control; (F) The half-life of TAB3 after treatment with actinomycin D for indicated times, with Lnc-PCIR knockdown or overexpression; (G, H) Co-IP assay to detect the association between TAB3 and PABPC4; (I) Western blot to analysis the interaction of PABPC4 with biotinylated-TAB3 mRNA using RNA pull-down samples in 231 cell lines. All $p$-values calculated by independent sample t-test $\left({ }^{\star \star *}\right.$ significant values of $\left.<0.001\right)$. ns, no significance.

(TGF- $\beta$ activated kinase 1) which implicated in regulating diverse range of cellular processes that include embryonic development, differentiation, autophagy, apoptosis and cell survival $(18,22)$. TAB3, a scaffold protein of TAB2, is involved in IL-1 and TNF- $\alpha$ signaling pathways (21). Several reports demonstrated TAB3 is widely expressed and constitutively overexpressed in certain tumor tissues, which as the oncogene to driving the occurrence and development of tumors $(24,33)$. Here, our findings showed that Lnc-PCIR have a strong impact on TAB3 mRNA stability, and interacting with and upregulating the TAB3 protein level which contribute to the promoting effects on activating TNF- $\alpha / N F-\kappa B$ pathway in TNBC cells. Next, we further explored the mechanisms that stabilize TAB3 mRNA by Lnc-PCIR. 
A

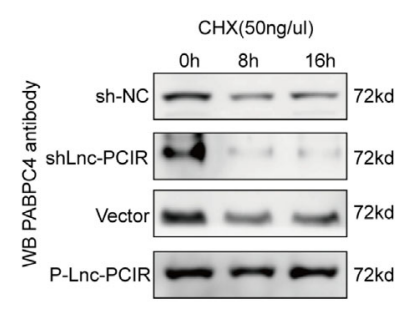

D

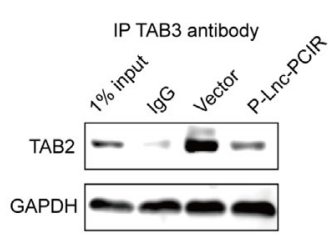

F

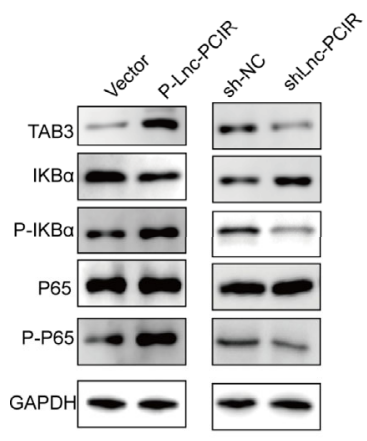

G
B

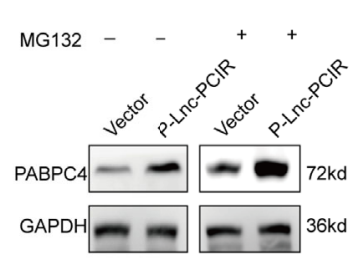

C

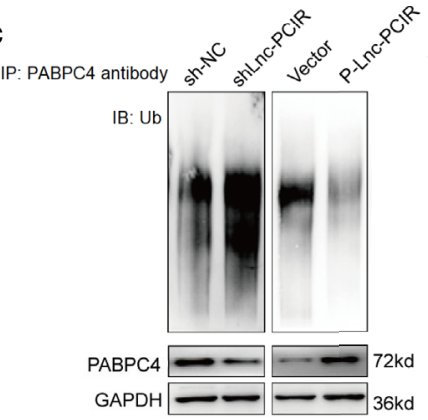

E
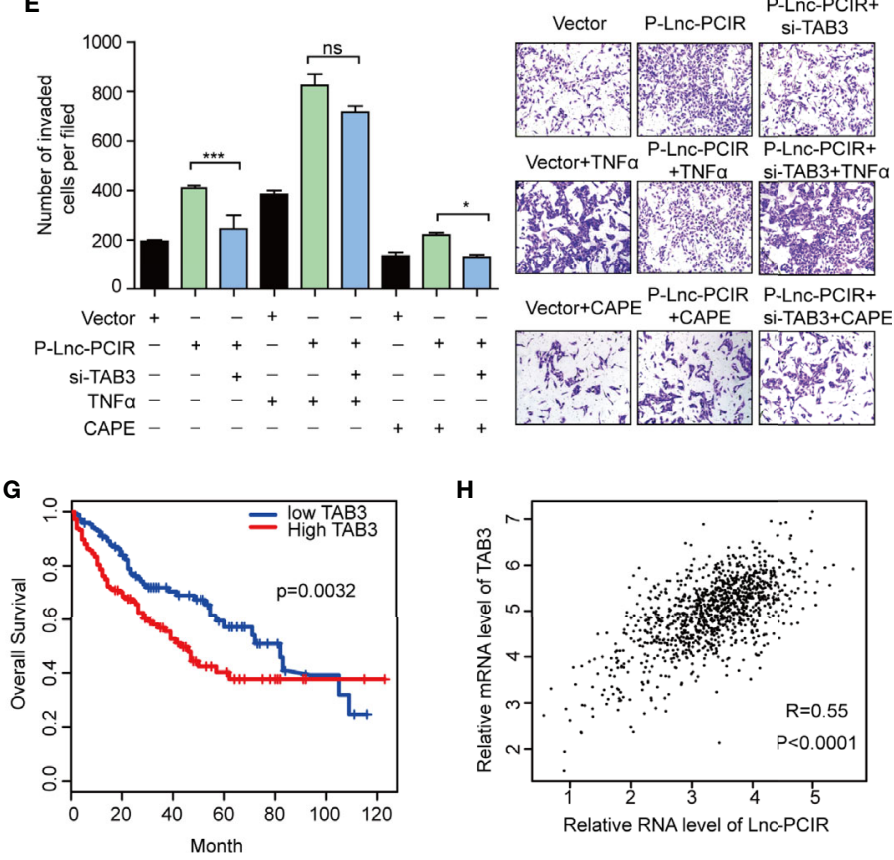

H

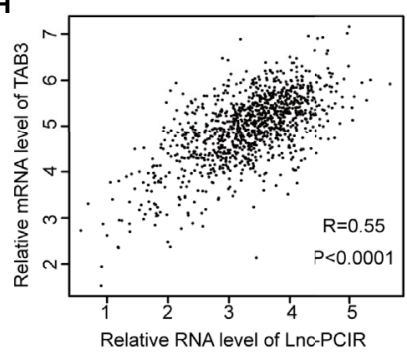

FIGURE 7 | Lnc-PCIR blocks proteasome-dependent ubiquitination degradation of PABPC4. (A) Western blot to detect the protein level of PABPC4 in sh-LncPCIR, P-Lnc-PCIR or the control cells which treated with CHX for the indicated times; (B) Western blot to detect the protein level of PABPC4 in P-Lnc-PCIR or control cells were treated with MG132 for 12 h; (C) IP assay with either control IgG or endogenous PABPC4 antibody and immunoblotted with the ubiquitin-specific antibody, endogenous PABPC4 and GAPDH served as the loading control; (D) Co-IP assay to detect the association between TAB3 and TAB2 after Lnc-PCIR overexpression, GAPDH served as the loading control; (E) Rescue assays of transwell invasion assay were performed after silencing TAB3 in P-Lnc-PCIR cells with or without TNF $\alpha /$ CAPE treatment; (F) Protein expression levels of TAB3, IKBa, p-IKBa, P65 and p-P65 transfected with P-Lnc-PCIR and vector in 231 cells;

(G) Kaplan-Meier survival analysis of TAB3 expression in TNBC patients $(n=110)$; $(\mathbf{H})$ Correlation analysis between TAB3 and Lnc-PCIR in TNBC patients $(n=110)$. All $p$-values calculated by independent sample t-test $\left({ }^{\star *}\right.$ significant values of $\left.<0.01\right)$. ns, no significance.

PABPC4, another associated protein of Lnc-PCIR, which has been demonstrated related to the inflammatory biomarker (Creactive protein) and anti-hepatitis $\mathrm{C}$ response, is expressed at a higher level in tumor tissues $(34,35)$. However, the mechanisms underlying the upregulated protein level of PABPC4 remain unclear. In the present study, we provided a new regulatory mechanism for PABPC4 via Lnc-PCIR. Higher level of LncPCIR blocks PABPC4 proteasome-dependent ubiquitination degradation to promote the oncogenic effects of PABPC4 on carcinogenesis of TNBC. Stable and highly expressed PABPC4 can further increase the stability of TAB3 mRNA, and the association of PABPC4 and TAB3 can disrupt the binding of $\mathrm{TAB} 3$ and $\mathrm{TAB} 2$ to activate the TNF- $\alpha / \mathrm{NF}-\kappa \mathrm{B}$ pathway.

\section{CONCLUSION}

This study identifies clinically relevant lncRNA Lnc-PCIR using a large cohort of samples from TCGA and identified it have prognostic value and may be directly implicated in the oncogenic phenotype. Our results show that Lnc-PCIR acts as an oncogene 
and significantly promotes TNBC cells tumorigenicity and metastasis in vitro and in vivo. Mechanistically, higher levels of Lnc-PCIR inhibits PABPC4 ubiquitination and degradation. Moreover, higher $\mathrm{PABPC} 4$ protein level strengthen the interaction of PABPC4 and TAB3 mRNA, weakens the interaction between $\mathrm{TAB} 3$ and $\mathrm{TAB} 2$, positively regulated TNF- $\alpha / N F-\kappa B$ signaling pathway. Therefore, our study implies the Lnc-PCIR may provide a potential target for TNBC treatment.

\section{DATA AVAILABILITY STATEMENT}

The original contributions presented in the study are included in the article/Supplementary Material. Further inquiries can be directed to the corresponding author.

\section{ETHICS STATEMENT}

The studies involving human participants were reviewed and approved by Shanghai Cancer Center of Fudan University. The patients/participants provided their written informed consent to participate in this study. The animal study was reviewed and approved by Fujian Medical University.

\section{REFERENCES}

1. DeSantis CE, Ma J, Gaudet MM, Newman LA, Miller KD, Goding Sauer A, et al. Breast Cancer Statistics, 2019. CA: Cancer J Clin (2019) 69:438-51. doi: 10.3322/caac. 21583

2. Bray F, Ferlay J, Soerjomataram I, Siegel RL, Torre LA, Jemal A. Global Cancer Statistics 2018: GLOBOCAN Estimates of Incidence and Mortality Worldwide for 36 Cancers in 185 Countries. CA: Cancer J Clin (2018) 68:394424. doi: $10.3322 /$ caac. 21492

3. Cancer Genome Atlas N. Comprehensive Molecular Portraits of Human Breast Tumours. Nature (2012) 490:61-70. doi: 10.1038/nature11412

4. Garrido-Castro AC, Lin NU, Polyak K. Insights Into Molecular Classifications of Triple-Negative Breast Cancer: Improving Patient Selection for Treatment. Cancer Discov (2019) 9:176-98. doi: 10.1158/2159-8290.CD-18-1177

5. Lehmann BD, Bauer JA, Chen X, Sanders ME, Chakravarthy AB, Shyr Y, et al. Identification of Human Triple-Negative Breast Cancer Subtypes and Preclinical Models for Selection of Targeted Therapies. J Clin Invest (2011) 121:2750-67. doi: 10.1172/JCI45014

6. Carey LA, Dees EC, Sawyer L, Gatti L, Moore DT, Collichio F, et al. The Triple Negative Paradox: Primary Tumor Chemosensitivity of Breast Cancer Subtypes. Clin Cancer Res (2007) 13:2329-34. doi: 10.1158/1078-0432.CCR-06-1109

7. Prensner JR, Chinnaiyan AM. The Emergence of lncRNAs in Cancer Biology. Cancer Discov (2011) 1:391-407. doi: 10.1158/2159-8290.CD-11-0209

8. Quinn JJ, Chang HY. Unique Features of Long non-Coding RNA Biogenesis and Function. Nat Rev Genet (2016) 17:47-62. doi: 10.1038/nrg.2015.10

9. Li Z, Lu X, Liu Y, Zhao J, Ma S, Yin H, et al. Gain of LINC00624 Enhances Liver Cancer Progression by Disrupting the HDAC6-TRIM28-ZNF354C Corepressor Complex. Hepatology (2020). doi: 10.1002/hep.31530

10. Gupta RA, Shah N, Wang KC, Kim J, Horlings HM, Wong DJ, et al. Long non-Coding RNA HOTAIR Reprograms Chromatin State to Promote Cancer Metastasis. Nature (2010) 464:1071-6. doi: 10.1038/nature08975

11. Tsai MC, Manor O, Wan Y, Mosammaparast N, Wang JK, Lan F, et al. Long Noncoding RNA as Modular Scaffold of Histone Modification Complexes. Science (2010) 329:689-93. doi: 10.1126/science.1192002

\section{AUTHOR CONTRIBUTIONS}

CW, WG, and $\mathrm{HH}$ designed the study. WG, JL, HH, and FF performed the experiments. WG and $\mathrm{HH}$ analyzed the results. YL carried out bioinformatics analyses. WG, $\mathrm{HH}$, and CW wrote the paper with comments from all authors. All authors contributed to the article and approved the submitted version.

\section{FUNDING}

This work was supported by grants from Joint funds for the innovation of science and Technology of Fujian province (2018Y9019, 2018Y9055), Joint funds for the youth research project of Fujian provincial health department (2011-1-14) and minimally invasive medicine center of Fujian Province.

\section{SUPPLEMENTARY MATERIAL}

The Supplementary Material for this article can be found online at: https://www.frontiersin.org/articles/10.3389/fonc.2021. 630300/full\#supplementary-material

12. Niknafs YS, Han S, Ma T, Speers C, Zhang C, Wilder-Romans K, et al. The lncRNA Landscape of Breast Cancer Reveals a Role for DSCAM-AS1 in Breast Cancer Progression. Nat Commun (2016) 7:12791. doi: 10.1038/ncomms12791

13. Pikarsky E, Porat RM, Stein I, Abramovitch R, Amit S, Kasem S, et al. NFKappab Functions as a Tumour Promoter in Inflammation-Associated Cancer. Nature (2004) 431:461-6. doi: 10.1038/nature02924

14. Caamano J, Hunter CA. NF-Kappab Family of Transcription Factors: Central Regulators of Innate and Adaptive Immune Functions. Clin Microbiol Rev (2002) 15:414-29. doi: 10.1128/CMR.15.3.414-429.2002

15. Tornatore L, Thotakura AK, Bennett J, Moretti M, Franzoso G. The Nuclear Factor Kappa B Signaling Pathway: Integrating Metabolism With Inflammation. Trends Cell Biol (2012) 22:557-66. doi: 10.1016/ j.tcb.2012.08.001

16. Ding J, Zhao J, Huan L, Liu Y, Qiao Y, Wang Z, et al. Inflammation-Induced Long Intergenic Noncoding Rna (Linc00665) Increases Malignancy Through Activating the Double-Stranded Rna-Activated Protein Kinase/Nuclear Factor Kappa B Pathway in Hepatocellular Carcinoma. Hepatology (2020) 72 (5):1666-81. doi: 10.1002/hep.31195

17. Jin X, Ding D, Yan Y, Li H, Wang B, Ma L, et al. Phosphorylated RB Promotes Cancer Immunity by Inhibiting NF-Kappab Activation and PD-L1 Expression. Mol Cell (2019) 73:22-35.e26. doi: 10.1016/j.molcel.2018.10.034

18. Sakurai H, Miyoshi H, Mizukami J, Sugita T. Phosphorylation-Dependent Activation of TAK1 Mitogen-Activated Protein Kinase Kinase Kinase by TAB1. FEBS Lett (2000) 474:141-5. doi: 10.1016/S0014-5793(00)01588-X

19. Cheung PC, Nebreda AR, Cohen P. TAB3, a New Binding Partner of the Protein Kinase TAK1. Biochem J (2004) 378:27-34. doi: 10.1042/bj20031794

20. Ishitani T, Takaesu G, Ninomiya-Tsuji J, Shibuya H, Gaynor RB, Matsumoto K. Role of the TAB2-related Protein TAB3 in IL-1 and TNF Signaling. EMBO $J$ (2003) 22:6277-88. doi: 10.1093/emboj/cdg605

21. Kim SI, Kwak JH, Na HJ, Kim JK, Ding Y, Choi ME. Transforming Growth Factor-Beta (TGF-beta1) Activates TAK1 Via TAB1-mediated Autophosphorylation, Independent of TGF-beta Receptor Kinase Activity in Mesangial Cells. J Biol Chem (2009) 284:22285-96. doi: 10.1074/jbc.M109.007146 
22. Jin G, Klika A, Callahan M, Faga B, Danzig J, Jiang Z, et al. Identification of a Human NF-kappaB-activating Protein, TAB3. Proc Natl Acad Sci USA (2004) 101:2028-33. doi: 10.1073/pnas.0307314101

23. Ding J, Huang S, Wang Y, Tian Q, Zha R, Shi H, et al. Genome-Wide Screening Reveals That miR-195 Targets the TNF-alpha/NF-kappaB Pathway by Down-Regulating IkappaB Kinase Alpha and TAB3 in Hepatocellular Carcinoma. Hepatology (2013) 58:654-66. doi: 10.1002/hep.26378

24. Chen J, Gu J, Feng J, Liu Y, Xue Q, Ni T, et al. TAB3 Overexpression Promotes Cell Proliferation in non-Small Cell Lung Cancer and Mediates Chemoresistance to CDDP in A549 Cells Via the NF-kappaB Pathway. Tumour Biol (2016) 37:3851-61. doi: 10.1007/s13277-015-3896-y

25. Parker JS, Mullins M, Cheang MC, Leung S, Voduc D, Vickery T, et al. Supervised Risk Predictor of Breast Cancer Based on Intrinsic Subtypes. J Clin Oncol (2009) 27:1160-7. doi: 10.1200/JCO.2008.18.1370

26. Sørlie T, Perou CM, Tibshirani R, Aas T, Geisler S, Johnsen H, et al. Gene Expression Patterns of Breast Carcinomas Distinguish Tumor Subclasses With Clinical Implications. Proc Natl Acad Sci USA (2001) 98:10869-74. doi: 10.1073/pnas.191367098

27. Kong L, Zhang Y, Ye ZQ, Liu XQ, Zhao SQ, Wei L, et al. CPC: Assess the Protein-Coding Potential of Transcripts Using Sequence Features and Support Vector Machine. Nucleic Acids Res (2007) 35:W345-9. doi: 10.1093/nar/gkm391

28. Lin MF, Jungreis I, Kellis M. PhyloCSF: A Comparative Genomics Method to Distinguish Protein Coding and non-Coding Regions. Bioinformatics (2011) 27:i275-282. doi: 10.1093/bioinformatics/btr209

29. Hsu CH, Hsu CW, Hsueh C, Wang CL, Wu YC, Wu CC, et al. Identification and Characterization of Potential Biomarkers by Quantitative Tissue Proteomics of Primary Lung Adenocarcinoma. Mol Cell Proteom (2016) 15:2396-410. doi: 10.1074/mcp.M115.057026

30. Liu D, Yin B, Wang Q, Ju W, Chen Y, Qiu H, et al. Cytoplasmic Poly(a) Binding Protein 4 is Highly Expressed in Human Colorectal Cancer and
Correlates With Better Prognosis. J Genet Genomics (2012) 39:369-74. doi: 10.1016/j.jgg.2012.05.007

31. Kanayama A, Seth RB, Sun L, Ea CK, Hong M, Shaito A, et al. TAB2 and TAB3 Activate the NF-kappaB Pathway Through Binding to Polyubiquitin Chains. Mol Cell (2004) 15:535-48. doi: 10.1016/j.molcel.2004.08.008

32. Du Z, Fei T, Verhaak RG, Su Z, Zhang Y, Brown M, et al. Integrative Genomic Analyses Reveal Clinically Relevant Long Noncoding RNAs in Human Cancer. Nat Struct Mol Biol (2013) 20:908-13. doi: 10.1038/ nsmb. 2591

33. Grimsey NJ, Lin Y, Narala R, Rada CC, Mejia-Pena H. Trejo J. G ProteinCoupled Receptors Activate P38 MAPK Via a non-Canonical TAB1-TAB2and TAB1-TAB3-dependent Pathway in Endothelial Cells. J Biol Chem (2019) 294:5867-78. doi: 10.1074/jbc.RA119.007495

34. Kini HK, Kong J, Liebhaber SA. Cytoplasmic Poly(a) Binding Protein C4 Serves a Critical Role in Erythroid Differentiation. Mol Cell Biol (2014) 34:1300-9. doi: 10.1128/MCB.01683-13

35. Yang H, Duckett CS, Lindsten T. iPABP, an Inducible Poly(a)-Binding Protein Detected in Activated Human T Cells. Mol Cell Biol (1995) 15:6770-6. doi: 10.1128/MCB.15.12.6770

Conflict of Interest: The authors declare that the research was conducted in the absence of any commercial or financial relationships that could be construed as a potential conflict of interest.

Copyright (C) 2021 Guo, Li, Huang, Fu, Lin and Wang. This is an open-access article distributed under the terms of the Creative Commons Attribution License (CC BY). The use, distribution or reproduction in other forums is permitted, provided the original author(s) and the copyright owner(s) are credited and that the origina publication in this journal is cited, in accordance with accepted academic practice. No use, distribution or reproduction is permitted which does not comply with these terms. 